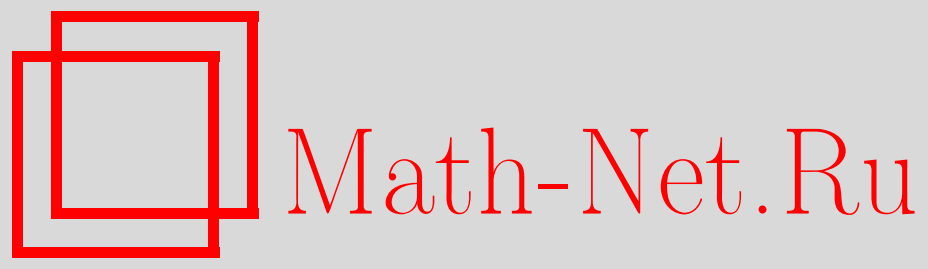

В. В. Лебедев, Об $l^{p}$-мультипликаторах функций, аналитических в круге, Функи. анализ и его прил., 2014, том 48, выпуск 3, 92-96

DOI: https://doi.org/10.4213/faa3159

Использование Общероссийского математического портала MathNet.Ru подразумевает, что вы прочитали и согласны с пользовательским соглашением

http://www . mathnet.ru/rus/agreement

Параметры загрузки:

IP : 54.198 .64 .247

26 апреля 2023 г., 12:19:49

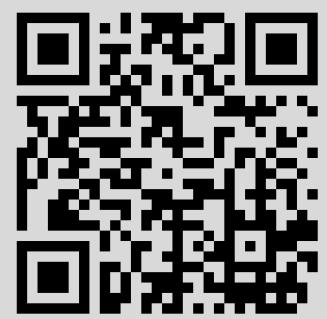




$$
q(x)=\frac{l(l+1)}{x^{2}}+\tilde{q}(x), \quad x \geqslant x_{0}, \quad \int_{x_{0}}^{\infty} x|\tilde{q}(x)| d x<\infty .
$$

(i) Если $l \in[-1 / 2,1 / 2)$, то $A$ подобен самосопряжсенному.

(ii) Eсли $l \geqslant 1 / 2$, то $A$ подобен самосопряженному в точности тогда, когда решение $c(x)$ неограничено.

Отметим, что случай $l=0$ исследован другим методом в [8, разд. 4].

Автор признателен М. М. Маламуду за многочисленные полезные обсуждения. Автор также благодарен рецензенту за критические замечания.

\title{
ЛитЕРАТУРА
}

[1] P. В. Акопян, Изв. АН Арм. ССР, Сер. матем., 15:5 (1980), 357-364. [2] R. Beals, J. Differential Equations, 56:3 (1985), 391-407. [3] C. Bennewitz, Proc. London Math. Soc., (3), 59:2 (1989), 294-338. [4] P. Binding, A. Fleige, Oper. Matrices, 5:4 (2011), 735755. [5] B. Ćurgus, A. Fleige, A. Kostenko, Integral Equations Operator Theory, 77:4 (2013), 533-557. [6] B. Ćurgus, H. Langer, J. Differential Equations, 79:1 (1989), 3161. [7] И. М. Карабаш, А. С. Костенко, Функц. анализ и его прил., 43:1 (2009), 81-84. [8] I. M. Karabash, A. S. Kostenko, M. M. Malamud, J. Differential Equations, 246:3 (2009), 964-997. [9] I. M. Karabash, M. M. Malamud, Oper. Matrices, 1:3 (2007), 301-368. [10] J. Korevaar, Tauberian Theory: A Century of Developments, Springer-Verlag, Berlin, 2004. [11] A. Kostenko, Math. Nachr., (2014) (to appear), DOI: 10.1002/mana.201300104; http://arxiv.org/abs/1202.2444. [12] H. Langer, in: Lect. Notes in Math., vol. 948, 1982, 1-46. [13] И. С. Кац, М. Г. Крейн, О спектралъных функииях струны. Доп. II $\kappa$ книге Ф. Аткинсона «Дискретные и непрерывные задачи», Мир, М., 1968. [14] А. И. Парфенов, Сиб. матем. журн., 44:4 (2003), 810-819. [15] С. Г. Пятков, в кн.: Неклассические уравнения математической физики, Изд-во Института математики СО РАН, Новосибирск, 2005, 240-251. [16] S. G. Pyatkov, in: Oper. Theory Adv. Appl., vol. 221, Birhäuser/Springer-Verlag, Basel, 2012, 549-570. [17] K. Veselić, Glasnik Math. Ser. III, 7:2 (1972), 229-248.

Faculty of Mathematics, University of Vienna e-mail: Oleksiy.Kostenko@univie.ac.at

Поступило в редакцию 11 июля 2012 г.

УДК $517.948+513.8$

\section{Об $l^{p}$-мультипликаторах функций, аналитических в круге*}

\author{
(C) 2014. В. В. ЛЕБЕДЕВ
}

Для произвольной функции $f$, аналитической в единичном круге $D=\{z \in$ $\mathbb{C}:|z|<1\}$ комплексной плоскости $\mathbb{C}$, рассмотрим ее разложение в ряд Тейлора:

$$
f(z)=\sum_{n \geqslant 0} \widehat{f}(n) z^{n}, \quad z \in D
$$

* Исследование осуществлено в рамках программы «Научный фонд НИУ ВШЭ» в 20132014 гг., проект №12-01-0079. 
При $1 \leqslant p \leqslant \infty$ пусть $A_{p}^{+}(D)$ - пространство всех функций $(1)$, таких, что последовательность коэффициентов Тэйлора $\widehat{f}=\{\widehat{f}(n), n=0,1, \ldots\}$ принадлежит $l^{p}$. Для $f \in A_{p}^{+}(D)$ положим $\|f\|_{A_{p}^{+}(D)}=\|\widehat{f}\|_{l^{p}}$. Аналитическая в $D$ функция $m$ называется $l^{p}$-мультипликатором, если для любой функции $f$ из $A_{p}^{+}(D)$ имеем $m \cdot f \in A_{p}^{+}(D)$. Класс всех таких мультипликаторов обозначим через $M_{p}^{+}(D)$. Этот класс является банаховой алгеброй относительно естественной нормы

$$
\|m\|_{M_{p}^{+}(D)}=\sup _{\|f\|_{A_{p}^{+}(D)} \leqslant 1}\|m \cdot f\|_{A_{p}^{+}(D)}
$$

и обычного умножения функций. Классы $M_{p}^{+}(D)$ изучались в работах [1]-[6] $]^{1)}$. Отметим, что случай $p \neq 1, \infty, 2$ представляет отдельный интерес. Хорошо известно, что $M_{p}^{+}(D)=M_{q}^{+}(D)$ при $1 / p+1 / q=1$ и

$$
A_{1}^{+}(D)=M_{1}^{+}(D)=M_{\infty}^{+}(D) \subseteq M_{p}^{+}(D) \subseteq M_{2}^{+}(D)=H^{\infty}(D),
$$

где $H^{\infty}(D)$ - пространство Харди ограниченных аналитических функций в $D$.

Пусть $\Omega \subseteq \mathbb{C}$ - открытая область, содержащая круг $D$. Мы укажем класс областей $\Omega$, таких, что всякая ограниченная аналитическая функция в $\Omega$ принадлежит $M_{p}^{+}(D)$. Случай, когда $\Omega$ содержит замыкание круга $D$, тривиален; в этом случае всякая ограниченная аналитическая функция в $\Omega$ принадлежит $A_{1}^{+}(D)$ и, следовательно, принадлежит $M_{p}^{+}(D)$ при всех $p, 1 \leqslant p \leqslant \infty$. Нетривиальным является случай, когда граница области $\Omega$ имеет общие точки с границей $\partial D=\{z \in \mathbb{C}:|z|=1\}$ круга $D$.

Виноградов [2] показал, что если $r>1,0 \leqslant \alpha<\pi / 2$ и $m$ - ограниченная аналитическая функция в области

$$
\Omega_{0}=\{z \in \mathbb{C}:|z|<r, \alpha<\arg (z-1)<2 \pi-\alpha\},
$$

то $m \in \bigcap_{1<p<\infty} M_{p}^{+}(D)$. С помощью этого результата Виноградов впервые указал примеры нетривиальных (т. е. бесконечных) произведений Бляшке в $M_{p}^{+}(D)$. Заметим, что граница всякой области $(2)$ имеет лишь одну общую точку с границей круга $D$ (а именно точку $z=1$ ). Как мы увидим, утверждение, аналогичное результату Виноградова, верно для областей значительно более широкого класса. Функции, аналитические в областях, рассматриваемых ниже, могут иметь несчетное множество особенностей на границе круга ${ }^{2)}$.

Как обычно, для произвольной области $\Omega \subseteq \mathbb{C}$ через $H^{\infty}(\Omega)$ будем обозначать пространство Харди ограниченных аналитических функций в $\Omega$. Для $g \in H^{\infty}(\Omega)$ полагаем $\|g\|_{H^{\infty}(\Omega)}=\sup _{z \in \Omega}|g(z)|$.

Пусть $J$ - дуга на граничной окружности $\partial D$, причем длина $|J|$ этой дуги строго меньше $\pi$. Пусть $T_{J}$ - произвольный открытый равнобедренный треугольник, основанием которого служит хорда, стягивающая дугу $J$, и боковые стороны которого лежат вне круга $D$. Обозначим через $\theta_{T_{J}}$ угол между окружностью $\partial D$ и боковой стороной треугольника $T_{J}$.

1) В §6 работы автора [6] имеется неточность. Вместо написанного там «интеграл Пуассона» должно быть «проекция Рисса $\sum_{k=-\infty}^{\infty} c_{k} e^{i k t} \rightarrow \sum_{k \geqslant 0} c_{k} z^{k} \gg$.

2) Попутно отметим, что в теореме Виноградова условие $\alpha<\pi / 2$ является существенным. Например, функция $S(z)=\exp \{(z+1) /(z-1)\}$ ограничена в полуплоскости $\{z \in \mathbb{C}: \operatorname{Re} z<1\}$, но, как показал Вербицкий [4], принадлежит $M_{p}^{+}(D)$ лишь в тривиальном случае $p=2$. 
Рассмотрим произвольное замкнутое множество $F \subseteq \partial D$. Пусть $\tau(F)-$ семейство всех дуг, дополнительных к $F$ (т. е. компонент связности дополнения $\partial D \backslash F)$. Будем предполагать, что каждая дуга семейства $\tau(F)$ имеет длину, строго меньшую, чем $\pi$. Рассмотрим область

$$
\Omega_{F}=D \cup \bigcup_{J \in \tau(F)} T_{J},
$$

причем потребуем выполнения условия

$$
\inf _{J \in \tau(F)} \theta_{T_{J}}>0 .
$$

Всякую область $\Omega_{F}$, полученную указанным образом, назовем звездообразной областью, порожденной множеством $F$.

Мы покажем, что при некотором условии, наложенном на множество $F \subseteq$ $\partial D$, всякая ограниченная аналитическая в $\Omega_{F}$ функция принадлежит $M_{p}^{+}(D)$.

Пусть $E$ - замкнутое множество лебеговой меры нуль на прямой $\mathbb{R}$. Рассмотрим семейство $\tau(E)$ всех интервалов, дополнительных к $E$ (т. е. компонент связности дополнения $\mathbb{R} \backslash E)$. Для произвольного интервала $I \subseteq \mathbb{R}$ определим оператор $S_{I}$, полагая

$$
\widehat{S_{I}(f)}=1_{I} \widehat{f}, \quad f \in L^{p} \cap L^{2}(\mathbb{R}),
$$

где означает преобразование Фурье и $1_{I}$ - характеристическая функция интервала $I$ (т. е. $1_{I}(t)=1$ при $t \in I, 1_{I}(t)=0$ при $t \notin I$ ). Следуя [7], скажем, что множество $E$ обладает свойством $\mathrm{LP}(p)(1<p<\infty)$, если соответствующая квадратичная функция Литтлвуда-Пэли

$$
S(f)=\left(\sum_{I \in \tau(E)}\left|S_{I}(f)\right|^{2}\right)^{1 / 2}
$$

удовлетворяет условию

$$
c_{1}(p)\|f\|_{L^{p}(\mathbb{R})} \leqslant\|S(f)\|_{L^{p}(\mathbb{R})} \leqslant c_{2}(p)\|f\|_{L^{p}(\mathbb{R})}, \quad f \in L^{p}(\mathbb{R})
$$

(с положительными константами $c_{1}(p), c_{2}(p)$, не зависящими от $f$ ). В случае, если $E$ обладает свойством $\mathrm{LP}(p)$ при всех $p, 1<p<\infty$, скажем, что $E$ обладает свойством LP.

Пусть теперь $F$ - замкнутое множество меры нуль на граничной окружности $\partial D$. Скажем, что $F$ обладает свойством $\mathrm{LP}(p)$ или свойством LP, если $F=\left\{e^{i t}, t \in E\right\}$, где $E \subseteq[0,2 \pi]-$ множество, обладающее свойством $\operatorname{LP}(p)$ или свойством LP соответственно.

Замечание 1. Классическим примером бесконечного множества $E \subseteq \mathbb{R}$, обладающего свойством LP, является $E=\left\{ \pm 2^{k}, k \in \mathbb{Z}\right\} \cup\{0\}$, где $\mathbb{Z}-$ множество целых чисел. Вместе с тем существуют несчетные множества, обладающие свойством LP. Впервые это было установлено Хеар и Клемешем [8]. Существование таких множеств также отмечалось в [9]; детали изложены в $[10, \S 4]$. Сформулируем соответствующий результат для множеств на $\partial D$. При каждом $p$, $1<p<\infty$, существует константа $\beta_{p}\left(0<\beta_{p}<1\right)$, такая, что верно следующее. Пусть $F \subseteq \partial D$ - замкнутое множество меры нуль. Предположим, что дуги $J_{k}$, $k=1,2, \ldots$, дополнительные к $F$, будучи занумерованы в порядке невозрастания их длин, удовлетворяют условию $\left|J_{k+1}\right| /\left|J_{k}\right| \leqslant \beta_{p}$ при всех достаточно 
больших $k$. Тогда $F$ обладает свойством LP $(p)$. Отсюда, в свою очередь, вытекает, что если $\lim _{k \rightarrow \infty}\left|J_{k+1}\right| /\left|J_{k}\right|=0$, то $F$ обладает свойством LP.

Результатом настоящей заметки является следующая

Теорема. Пусть множество $F \subseteq \partial D$ обладает свойством $\operatorname{LP}(p)$ u $\Omega_{F}-$ звездообразная область, порожденная $F$. Тогда $H^{\infty}\left(\Omega_{F}\right) \subseteq M_{p}^{+}(D)$. Eсли $F$ обладает свойством LP , то $H^{\infty}\left(\Omega_{F}\right) \subseteq \bigcap_{1<p<\infty} M_{p}^{+}(D)$.

Доказательство. Пусть $G$ - абелева группа и $\Gamma$ - группа, двойственная к $G$. Рассмотрим функцию $m \in L^{\infty}(\Gamma)$ и оператор $Q$, определяемый соотношением

$$
\widehat{Q f}=m \widehat{f}, \quad f \in L^{p} \cap L^{2}(G),
$$

где ^ означает преобразование Фурье на $G$. Функция $m$ называется $L^{p}$-мультипликатором Фурье, если соответствующий оператор $Q$ является ограниченным оператором в $L^{p}(G)(1 \leqslant p \leqslant \infty)$. Класс всех таких мультипликаторов обозначается через $M_{p}(\Gamma)$, при этом полагаем $\|m\|_{M_{p}(\Gamma)}=\|Q\|_{L^{p}(G) \rightarrow L^{p}(G)}$. Хорошо известна [11] (см. также [12]) связь между мультипликаторами Фурье на прямой $\mathbb{R}$ и на окружности $\mathbb{T}=\mathbb{R} / 2 \pi \mathbb{Z}$. Нам потребуется теорема Джодета $[12]$ о периодическом продолжении мультипликаторов. Согласно этой теореме, если $f \in M_{p}(\mathbb{R})$ - функция, аннулирующаяся вне отрезка $[0,2 \pi]$, и $g$ есть $2 \pi$-периодическая функция, совпадающая с $f$ на $[0,2 \pi]$, то $g \in M_{p}(\mathbb{T})$. Отметим, что между пространствами $M_{p}^{+}(D)$ и $M_{p}(\mathbb{T})$ имеется непосредственная связь. Имея произвольную функцию $m \in H^{\infty}(D)$, рассмотрим ее (некасательную) граничную функцию $m^{*}(t)=m\left(e^{i t}\right)$. Условия $m \in M_{p}^{+}(D)$ и $m^{*} \in M_{p}(\mathbb{T})$ эквивалентны [3] (см. также [5]).

Нам потребуется также следующее утверждение. Пусть множество $E \subseteq \mathbb{R}$ обладает свойством LP $(p)$. Предположим, что функция $f \in L^{\infty}(\mathbb{R})$ непрерывно дифференцируема на каждом интервале, дополнительном к $E$, и ее производная $f^{\prime}$ удовлетворяет условию

$$
\left|f^{\prime}(t)\right| \leqslant \frac{c}{\operatorname{dist}(t, E)}, \quad t \in \mathbb{R} \backslash E,
$$

где $\operatorname{dist}(t, E)$ означает расстояние от точки $t$ до множества $E$ и $c>0$ не зависит от $t$. Тогда $f \in M_{p}(\mathbb{R})$. Этот результат, принадлежащий Шёгрену и Шёлину [7], является обобщением хорошо известной теоремы Михлина-Хёрмандера.

Заметим теперь, что условие (3) влечет за собой существование константы $c=c\left(\Omega_{F}\right)>0$, такой, что если $e^{i t} \in \partial D \backslash F$, то окружность с центром в $e^{i t}$ и радиусом $r(t)=c \cdot \operatorname{dist}\left(e^{i t}, F\right)$ целиком содержится в $\Omega_{F}$. Обозначим эту окружность через $\gamma(t)$. Пусть $m \in H^{\infty}\left(\Omega_{F}\right)$. Рассмотрим произвольную дугу $J$, дополнительную к $F$. Пусть $e^{i t} \in J$. Рассмотрим соответствующую окружность $\gamma(t)$. Для произвольной точки $z$, лежащей внутри окружности $\gamma(t)$, имеем

$$
m^{\prime}(z)=\frac{1}{2 \pi i} \int_{\gamma(t)} \frac{m(\zeta)}{(\zeta-z)^{2}} d \zeta .
$$

В частности

$$
m^{\prime}\left(e^{i t}\right)=\frac{1}{2 \pi i} \int_{\gamma(t)} \frac{m(\zeta)}{\left(\zeta-e^{i t}\right)^{2}} d \zeta .
$$


Отсюда для производной $\left(m^{*}\right)^{\prime}$ граничной функции $m^{*}(t)=m\left(e^{i t}\right)$ получаем

$$
\begin{aligned}
\left|\left(m^{*}\right)^{\prime}(t)\right| & =\left|i e^{i t} m^{\prime}\left(e^{i t}\right)\right|=\left|\frac{1}{2 \pi i} \int_{\gamma(t)} \frac{m(\zeta)}{\left(\zeta-e^{i t}\right)^{2}} d \zeta\right| \leqslant \frac{1}{2 \pi} \int_{\gamma(t)} \frac{|m(\zeta)|}{\left|\zeta-e^{i t}\right|^{2}}|d \zeta| \\
& \leqslant \frac{1}{2 \pi} 2 \pi r(t)\|m\|_{H^{\infty}\left(\Omega_{F}\right)} \frac{1}{(r(t))^{2}}=c_{1}\left(\Omega_{F}\right)\|m\|_{H^{\infty}\left(\Omega_{F}\right)} \frac{1}{\operatorname{dist}\left(e^{i t}, F\right)} .
\end{aligned}
$$

Пусть $E \subseteq[0,2 \pi]$ - множество, такое, что $F=\left\{e^{i t}, t \in E\right\}$ и $E$ обладает свойством LP $(p)$. Не ограничивая общности, можем считать, что $E$ содержит точки 0 и $2 \pi$. Определим функцию $f$ на прямой $\mathbb{R}$, полагая $f(t)=1_{[0,2 \pi]}(t) m^{*}(t)$, $t \in \mathbb{R}$. Мы видим (см. (5)), что функция $f$ удовлетворяет условию (4). Следовательно, по теореме Шёгрена-Шёлина, имеем $f \in M_{p}(\mathbb{R})$. Отсюда, пользуясь теоремой Джодета, получаем $m^{*} \in M_{p}(\mathbb{T})$. В силу связи между мультипликаторами на $\mathbb{T}$ и мультипликаторами функций, аналитических в круге $D$, получаем, что $m \in M_{p}^{+}(D)$.

Замечание 2. Насколько известно автору, вопрос о существовании множеств, обладающих свойством $\mathrm{LP}(p)$ при каком-то $p, p \neq 2$, и не обладающих свойством LP, открыт.

\section{ЛитеРАТУРА}

[1] С. А. Виноградов, ДАН СССР, 254:6 (1980), 1301-1306. [2] С. А. Виноградов, Зап. научн. сем. ЛОМИ, 39 (1974), 30-39. [3] Н. К. Никольский, Сиб. матем. ж., 7 (1966), 146-158. [4] И. Э. Вербицкий, Функц. анализ и его прил., 14:3 (1980), 67-68. [5] В. В. Лебедев, Функц. анализ и его прил., 32:4 (1998), 10-21. [6] V. V. Lebedev, in: Oper. Theory Adv. Appl., vol. 113, 2000, 205-212. [7] P. Sjögren, P. Sjölin, Ann. Inst. Fourier, Grenoble, 31:1 (1981), 157-175. [8] K. Hare, I. Klemes, Trans. Amer. Math. Soc., 347:10 (1995), 4105-4127. [9] V. Lebedev, A. Olevskiĭ, C. R. Acad. Sci. Paris, Ser. I, 322 (1996), 143-147. [10] В. В. Лебедев, А. М. Олевский, Изв. РАН, сер. Матем., 70:3 (2006), 129-166. [11] K. de Leew, Ann. Math., 81 (1965), 364-379. Studia Math., 34 (1970), 215-226.

Национальный исследовательский университет «Высшая школа экономики»

Поступило в редакцию Московский институт электроники и математики 22 марта 2013 г. e-mail: lebedevhome@gmail.com

Заведующая редакцией и научный редактор Г. М. Цукерман

Подписано к печати 24.07.2014. Дата выхода в свет 20.08.2014. Формат 70×100/16 Печать офсетная. Усл. печ. л. 7,8. Усл. кр.-отт. 1,4 тыс. Бум. л. 3,0 Уч.-изд. л. 8,0. Тираж 172 экз. Заказ 374.

Учредители: Российская академия наук, Математический институт им. В. А. Стеклова РАН

Адрес редакции: 117966 Москва, ГСП-1, ул. Губкина 8, комн. 624. Тел. 938-37-56

Издатель: Российская академия наук, Издательство «Наука»,

Отпечатано в ППП «Типография «Наука», 121099 Москва, Шубинский пер., 6 